\title{
The mathematical model of gas flowing in porous medium based on the homogenization method
}

\author{
Wei Xiong ${ }^{1,2 *}$ \\ ${ }^{1}$ China Coal Technology and Engineering Group Chongqing Research Institute, Chongqing 400037, China \\ ${ }^{2}$ State Key Laboratory of the Gas Disaster Detecting, Preventing and Emergency Controlling, Chongqing 400037, China
}

\begin{abstract}
Considered the characteristics of porous medium in the coal seam and goaf, in order to reflect the accurately influence of various porous media against the gas flow, the mathematical model of discrete multiscale network and macroscopic flow, CFCM (Coal-Fracture-Cavity-Model), was presented. The porous medium is classified into coal matrix, fracture and hole systems based on the size, and the coal matrix system includes micro fractures and micro-porous. The coal matrix system and fracture system can be regarded as diffusion and percolation areas; hole system can be regarded as a free-flowing area. The computation model of flow field in micro-scale, small-scale and large-scale are obtained according the Fick's diffusion law, Darcy's permeability law and Forchheimer generalized Darcy law respectively, the homogenization method is used to analyse the mathematical model by scale upgrading and the equivalent Darcy's fluid equation of porous medium is got to describe the characteristics of the medium in the flow field accurately. An example calculated shows that the coal matrix and fracture systems are the most influential factors of the flow field in goaf and the two systems above would prevent the diffusion of airflow. The study validates the correctness of the classification method and the model of flow equation.
\end{abstract}

\section{Introduction}

Mastering the law of gas migration and accumulation in coal seams, fractured rock masses and goafs are the basis for the development of coal mine gas prevention and drainage technology[1]. In 1947, Soviet scholar P.M. Krichevsky[2] used the theory of permeability to describe the process of gas migration in coal seams, and obtained the law of gas seepage considering the nature of gas adsorption. A.Saghfi and R.J. William[3] studied the laws of adsorption and free gas migration in coal, and found that the permeability of coal is closely related to the fracture network. Liang and Zhang[4] proposed to treat gas flow as the flow of compressible fluid in a deformable solid framework, and obtained a coupled mathematical model of gas flow in coal and rock layers under the influence of mining. Based on the theory of porous media seepage and dynamic dispersion, Qi[5] established a three-dimensional stable seepage and gas concentration distribution mathematical model, and gave the Galerkin finite element solution of the mathematical model.

It reveals the law of gas flow in coal to a certain extent by the above-mentioned research work, but they are all limited to one medium system and fail to fully and accurately reflect the gas flow characteristics in porous media. The law of gas migration by coal matrix, cracks, and hole systems has not been explained in a unified manner. In this paper, for the special system of porous media, a CFCM (Coal-Fracture-Cavity-Model) model is proposed to accurately describe the flow characteristics of gas in the mine. The equivalent Darcy flow equation of large-scale space is derived based on the homogenization theory, and the simulation software is used to analyze the case to verify the correctness of the model.

\section{The multiscale medium and CFCM model}

$10 \sim 20 \%$ of the gas which in the original coal exist in the cracked holes in free form or be adsorbed on the surface of the coal mass, and about $80 \%$ of gas is attached to the molecular surface of the coal mass in an adsorbed state[6]. When the coal rock mass is exposed to the mining operation space, the high-pressure gas in the original coal and rock mass is decompressed and desorbed, and the free and desorbed gas migrates to the low-pressure space along the holes or fracture channels. With the influence of coal seam mining, stope area increase, overlying rock movement, etc., the rock strata began to loosen and deform, evolving from micro-holes into cracks and holes, forming pressure relief gas flow channels and a crack system for gas accumulation. As the coal seam is further mined, the rock straddles and forms a goaf. The remaining coal and roof falling rocks in the goaf are accumulated and recompacted, which can be regarded as a porous medium. This system is called a hole system.

Due to the different effects of diagenesis and its epigenesis, mining effects, and rock fall on coal and rock

\footnotetext{
"Corresponding author's e-mail: 2013163@cqccteg.com, xiongwei_sczg@163.com
} 
formations, multi-scale media systems have great differences in hole space morphology, distribution, and occurrence. The main characteristics are as follows[7] :

(1) The hole space has various forms, including coal matrix, fractures and holes. Coal matrix includes coal body, micro-cracks and micro-pores, which have many genesis and types, the porosity and permeability of the coal matrix is low. The three-dimensional spatial scale of the fracture system is quite different, and the ratio of the smaller one to the other larger two-dimensional holes is less than 1/10. The hole system refers to the holes with similar threedimensional spatial scale and no less than $2 \mathrm{~cm}$, which is regarded as a free flow space of gas.

(2) The size of the hole space varies greatly, and the spatial scale ranges from a few microns to a few meters, which has a strong heterogeneity.

The above characteristics indicate that the multi-scale media system is actually a discrete fracture and hole network space. The flow has both porous media seepage and free flow in large space, and it is a complex coupled flow system. The existing seepage theories (such as dualmedium and multi-medium theories) can no longer effectively describe its flow characteristics. Therefore, this paper proposes a multi-scale network (CFCM) flow mathematical model of coal matrix, fractures and holes, aiming to accurately describe the flow of gas in coal seams and goafs.

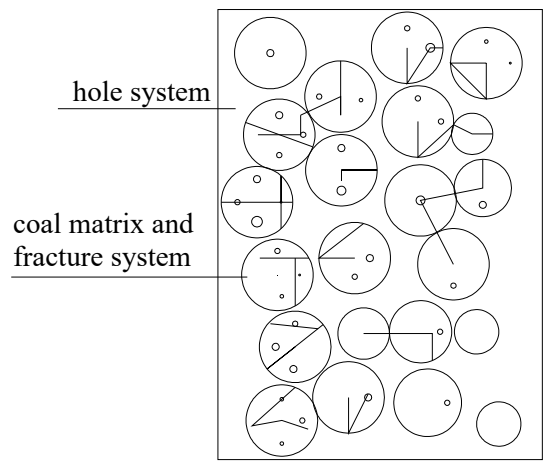

Figure 1. The model of CFCM

The CFCM model divides the coal body into a coal matrix system, a fracture system, and a hole system. The coal matrix system and the fracture system are regarded as the diffusion-seepage area, and the hole system is regarded as the free flow area. The coal body can be divided into micro-scale, small-scale and large-scale.

\section{CFCM Macro Flow Model}

Define $K(r, L)$ as a cube with a side length of $\mathrm{L}$ inside the porous medium centered on point $\mathrm{r}$, then $K(r, L)$ actually defines a measurement unit, and the porosity of the measurement unit $K(r, L)$ is[8]:

$$
\varphi(\mathrm{r}, L)=\frac{V(P \bigcap K(\mathrm{r}, L))}{V(K(r, l))}
$$

The general form of the governing equation is:

$$
\frac{\partial(\varphi \rho \phi)}{\partial t}+\frac{\partial(\varphi \rho u \phi)}{\partial x}+\frac{\partial(\varphi \rho v \phi)}{\partial y}+\frac{\partial(\varphi \rho w \phi)}{\partial z}=\frac{\partial}{\partial x}\left(\Gamma \frac{\partial \phi}{\partial x}\right)+\frac{\partial}{\partial y}\left(\Gamma \frac{\partial \phi}{\partial y}\right)+\frac{\partial}{\partial x}\left(\Gamma \frac{\partial \phi}{\partial y}\right)+S
$$

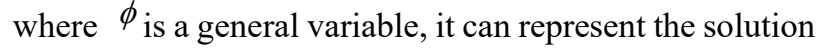
variables such as speed, temperature, component concentration, etc. $\Gamma$ is the generalized diffusion coefficient and $S$ is Generalized the source term. The items of Equ. (1) are transient, convection, diffusion, and source in order. And porosity ( $\varphi$ ) has the following relationship with permeability $(\mathrm{K})$ :

$$
K=C_{K} \frac{\varphi^{3}}{(1-\varphi)^{2}}, \quad K=\left\{\begin{array}{l}
K_{\mathrm{m}} \\
K_{\mathrm{f}} \\
K \mathrm{v}
\end{array}\right.
$$

where the $K_{\mathrm{m}}$ is the permeability of the coal matrix system, the $K_{\mathrm{f}}$ is the permeability of the fracture system, the $K_{\mathrm{v}}$ is the permeability of the hole system.

\subsection{Coal matrix and fracture system}

The hole distribution and fracture system in the coal body and related studies show that the flow of each component gas in the small scale is mainly diffusion movement and seepage movement. The diffusion movement basically obeys the Fick diffusion law and mainly occurs in the micro-holes of the coal body. The seepage movement obeys Darcy's law of permeability, which mainly occurs in the macro-holes and micro-cracks of coal. Therefore, the movement of component gas in the micro-scale and small- scale can be considered as a diffusion-percolation process.

For each component gas, the net flow produced by the system is the flow produced by system adsorption or desorption, and the concentration diffusion formula of the component gas is:

$$
\frac{\partial\left(\varphi C_{\mathrm{s}}\right)}{\partial \mathrm{t}}+\nabla\left(C_{\mathrm{s}} V\right)+\nabla J=S
$$

where the $\varphi$ is porosity; ${ }^{C_{\mathrm{s}}}$ is the concentration of each component gas and $J$ is the diffusion flow rate of each component gas.

The gas flow state of micro-scale and small-scale systems satisfies Darcy's law of permeability, then:

$$
V=-K(\nabla P)
$$

\subsection{Hole system}

The flow velocity of the fluid in the hole system increases with the increase of the scale. The pressure drop in the characteristic porous medium is the flow with a non-linear relationship between flow rates obeys Ahmeed Sunada (Forchheimer relationship). The seepage system is nonlinear. This non-linear relationship can be described by Forchheimer's generalized Darcy law[9]:

$$
\nabla P=-\frac{\mu}{K} V-\frac{C_{F}}{\sqrt{K}} \rho|V| V
$$

where $C_{F}$ is the inertia coefficient of fluid inertia effect. 
When the gas components flow in the system, the energy caused by the shear action cannot be ignored when the speed is fast enough, and the shear force of the viscous fluid needs to be considered. In Brinkman's generalized Darcy's law, if we consider the influence of inertia and combine the Forchheimer equation, we can get the B-F generalized Darcy's law that considers the overall resistance of porous media (Darcy resistance), inertial resistance and boundary layer viscous resistance[10]:

$$
\nabla P=-\frac{\mu}{K} V-\frac{C_{F}}{\sqrt{K}} \rho|V| V-\beta \frac{\mu}{\varphi} \nabla^{2} V
$$

For the hole system, the gas flow state satisfies the BF generalized Darcy's law, and the velocity $(V)$ is given by Equ. (5).

\subsection{Unity of the two systems}

The unified equations for coal matrix, fracture system and hole system are:

$$
\left\{\begin{array}{l}
\nabla P=-\frac{\mu}{K} V-\alpha \frac{C_{F}}{\sqrt{K}} \rho|V| V-\beta \frac{\mu}{\phi} \nabla^{2} V \\
\nabla \cdot V=0
\end{array}\right.
$$

The first kind boundary condition ( $\left.\Gamma_{1}\right)$ satisfies
Dirichlet's law[11], the velocity or pressure distribution on the boundary is shown as:

$$
\left.v\right|_{\Gamma_{1}}=\bar{v},\left.\quad p\right|_{\Gamma_{1}}=\bar{p}
$$

The secend kind boundary condition $\left(\Gamma_{2}\right)$ satisfies Dirichlet's law[12], the flow distribution along the outer normal on the boundary is shown as:

$$
\left.\frac{\partial v(x, t)}{\partial n}\right|_{\Gamma_{2}}=q(x, t)
$$

\section{The homogenization theory}

The homogenization theory is also called equivalent medium theory[13]. For a certain composite material, due to the existence of heterogeneity, under the action of volume force $\left({ }^{f}\right)$ and surface force $(t)$, the change of stress or displacement in the field of macroscopic position $\mathrm{X}$ appears to be smooth (Fig. 2a). If the coordinate system $y=x / \varepsilon$ is introduced (equivalent to magnifying the macroscopic coordinate system infinite times, where $\varepsilon$ is a small amount that infinitely approaches zero), the macroscopic and mesoscopic properties of the material can be combined and studied together (Fig. 2b).

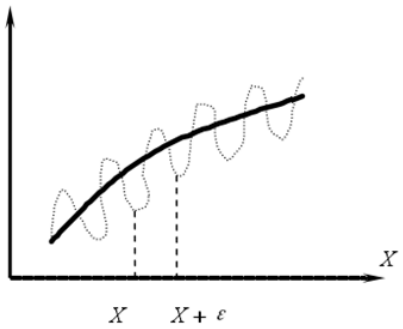

Figure 2(a). Macro function curves

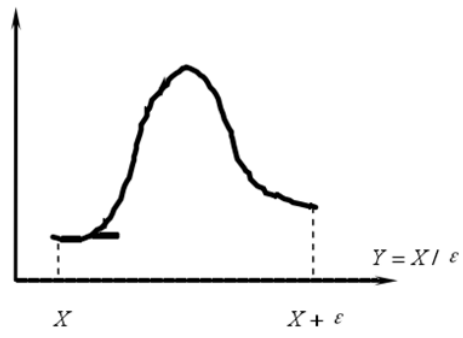

Figure 2(b). Local amplification

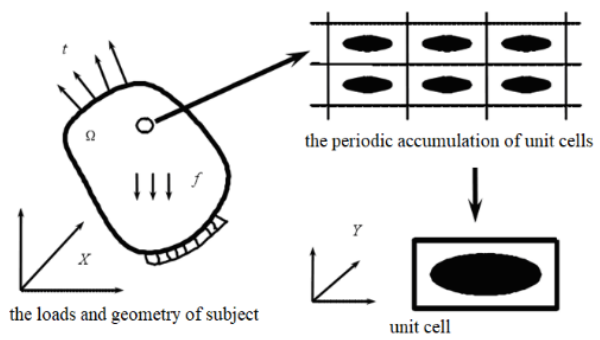

Figure 3. The schematic of homogenization theory
According to this feature of the homogenization theory, it is assumed that a certain point in the heterogeneous research body is infinitely enlarged[14]. It presents a mesoscopic structure formed by the accumulation of periodic unit cells. Select the mesoscopic coordinate system $\mathrm{Y}$ for a certain unit cell, so that the macroscopic (a certain point in the $\mathrm{X}$ coordinate system) and the mesoscopic (Y coordinate) A unit cell in the line): $f(x)=f(x, y)$.

The CFCM system is regarded as a fracture-porous medium with a $\mathrm{Y}$ periodic structure with closed boundaries. Assuming that there is a periodic medium area $(\Omega)$ in the goaf, and the entire boundary $(\Gamma)$ of this area includes the boundary of seepage-diffusion area $\left(\Gamma_{d}\right)$ and the boundary of free flow area $\left(\Gamma_{s}\right)$. The fluid inertia effect $\left({ }^{\alpha} \frac{C_{F}}{\sqrt{K}} \rho\left|V^{\varepsilon}\right| V^{\varepsilon}\right)$ is related to the density of the fluid and can be expressed as $\rho f^{\varepsilon}$, where $f$ is unit mass force. The gas flow model in the goaf is as follows:

$$
\left\{\begin{array}{l}
\nabla P^{\varepsilon}=-\frac{\mu}{K^{\varepsilon}} V^{\varepsilon}-\rho f^{\varepsilon}-\beta \frac{\mu}{\varphi} \varepsilon^{2} \nabla^{2} V^{\varepsilon} \\
\nabla \cdot V^{\varepsilon}=0
\end{array}\right.
$$

In order to satisfy the existence of physical limit when $\varepsilon \rightarrow 0$, both $\mathrm{K}$ and $\mu$ are multiplied by the scale factor $\varepsilon^{2}$

where $w_{j}$ and $\pi_{j}$ both are the function with Y period which are also about y, and they can be obtained by solving the following auxiliary problems:

$$
\begin{aligned}
& \frac{w_{j}}{K}-\beta \frac{\mu}{\varphi} \nabla_{y} w_{j}+\nabla_{y} \pi_{j}=e_{j} \\
& \nabla w_{j}=0
\end{aligned}
$$

However, the above auxiliary problems are difficult to obtain analytical solutions and need to be solved numerically. In the specific calculations, the standard Galerkin finite element method is first used to derive the seepage-diffusion zone and the free flow zone respectively, and then the unified flow pattern is obtained through the continuity equation with the finite element solution format.

\section{Case analysis}

In order to balance the contradiction between the calculation convergence and accuracy and the solution time, this paper uses the common free-division triangular mesh, and the open boundary CFCM model is shown in Fig. 4. The permeability of the homogeneous rock block in the hole space is $1.44 \times 10^{-7} \mathrm{~m}^{2}$, and the porosity of it is 0.9 . The permeability of the coal matrix and the isotropic 
medium in the fissure space is $6.42 \times 10^{-8} \mathrm{~m}^{2}$ and the porosity is 0.2 . The gas fluid density $(\rho)$ and viscosity are $0.714 \mathrm{~kg} / \mathrm{m}^{3}$ and $1.87 \times 10^{-5} \mathrm{~Pa} \cdot \mathrm{S}$ respectively. The upper and lower boundaries and coal-falling gas replenishment volume are ranging from $1 \times 10^{-4} \sim 1 \times 10^{-6} \mathrm{~mol} / \mathrm{m}^{2} \cdot \mathrm{s}$. The

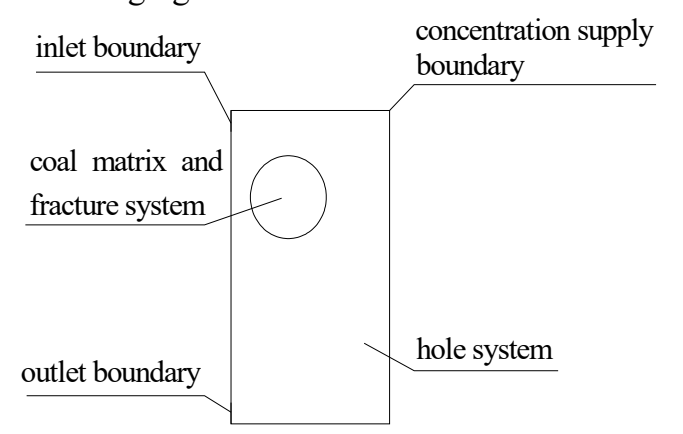

Figure 4. The schematic of calculating model

The arrow in the velocity distribution diagram (Fig. 5) indicates the direction of the velocity, and the colour indicates the magnitude of the velocity. It is not difficult to see from the figure that the velocity of the air flow decreases after entering the coal matrix and fracture system, and turbulence is formed around the coal matrix and fracture system.

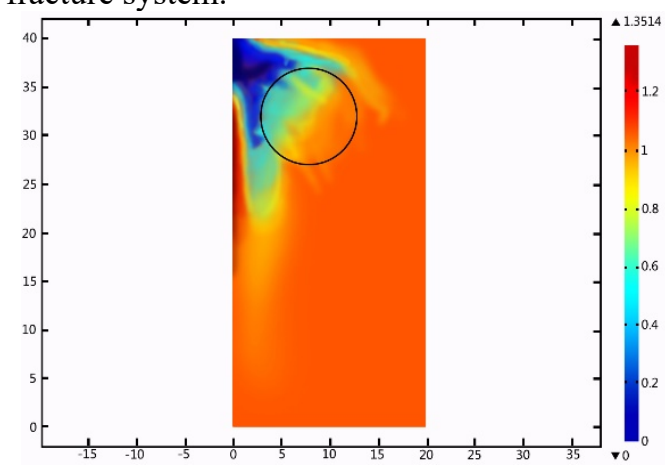

Figure 6. The distribution of gas at 100s

It can be seen from the above calculation examples that the CFGM model can effectively describe the basic flow characteristics of the goaf system:

(1) Flow mainly occurs in the hole system, and the flow is weak in the coal matrix and fracture system.

(2) The coal matrix and fissure system prevent the diffusion of wind current, and its own gas concentration is relatively high in a short time.

(3) The boundary of coal matrix, fracture system and hole system will cause turbulence of wind flow.

(4) The existence of coal matrix and fracture system causes the heterogeneity of the mined-out area system is also obvious: the flow velocity in the hole system is much greater than that in the coal matrix and fracture system.

\section{Conclusion}

(1) According to the characteristics of porous media in coal and rock mass, we propose a CFCM macromathematical flow model. The equivalent Darcy flow equation is derived and the solution formula for the porous media flow field is derived based on the homogenization upper $5 \mathrm{~m}$ on the left is the inlet boundary, and the lower $5 \mathrm{~m}$ on the left is the outlet boundary. The inlet and outlet pressure difference is $200 \mathrm{~Pa}$, and the other boundaries are airtight boundaries.

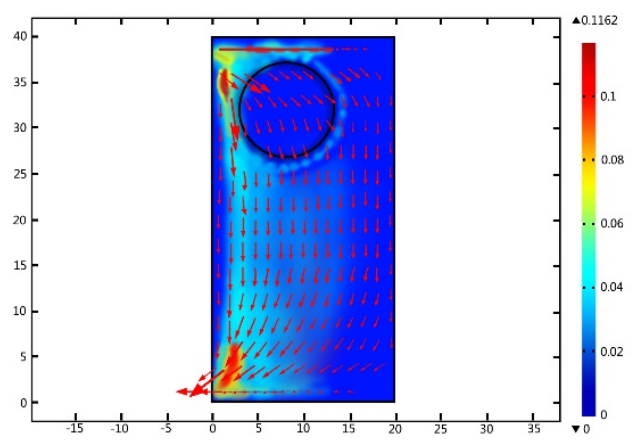

Figure 5. The speeding schematic of calculating model

The gas concentration distribution at $100 \mathrm{~s}$ and $200 \mathrm{~s}$ is shown in Fig. 6 and Fig. 7, respectively. And the degree of lightness and darkness of the colour indicates the size of the gas concentration. We can get that the existence of coal matrix and fissure system hinders the diffusion of wind current, making its own gas concentration higher.

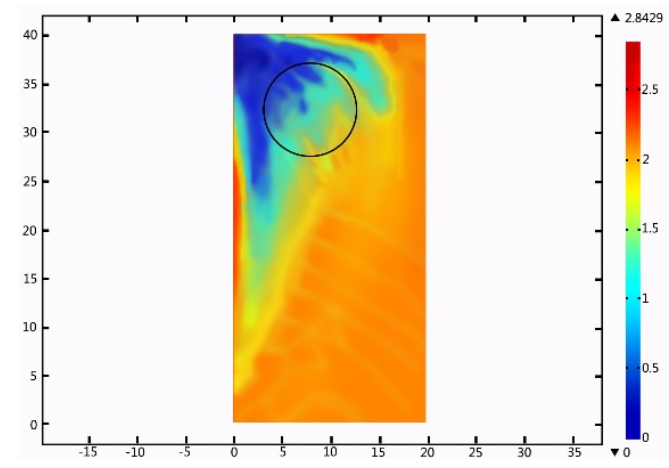

Figure 7. The distribution of gas at 200 s

theory.

(2) The analysis results of the calculation examples prove that the CFCM model can effectively describe the basic flow characteristics of the porous media in the goaf, and it is a new model with development prospects.

(3) Based the CFCM simulation results, we can get that the coal matrix and fracture system are particularly important for the flow field in the goaf, and the coal matrix and fracture system prevent the spread of wind current.

\section{Acknowledgments}

This work was financially supported by the Tiandi technology co. LTD Special funds for scientific and technological innovation and entrepreneurship (2019-TDQN038), the entrepreneurship of Chongqing Research Institute of CCTEG Crop.(2018ZDXM05), which are gratefully acknowledged.

\section{References}

1. Yu Q.X. Prevention and control of mine gas[M]. 
Xuzhou: China University of Mine and Technology Press, 1992.

2. A.T. Aruny. The Prediction and Prevention of Coal Mine Gas Dynamic Phenomena[M] Beijing: Coal Industry Press. 1992

3. Saghafi. A, Computer simulation of gas flow in coal mine and its application in the prediction of gas emission and gas drainage[C].22nd international mining safety conference proceedings. Beijing: Coal Industry Press, 1987.

4. Liang B, Zhang M.T. The numerical simulation on the seepage law of compressible gas in coal seams[C].North of China rock mechanics and engineering applications conference proceedings. Beijing: Science Press, 1991.

5. Qi Q.J, Feng S.H, etc. The mathematical model of three-dimensional steady seepage and gas distribution in stope and its finite element solution[J] , Journal of Liaoning Technical University. 1990,9(3):39 46.

6. Zhou S.N, Lin B.Q. The Theory of Gas Flow and Storage in Coal Seams, China Coal Industry Press.1998.

7. C, Meile, K, Tuncay. Scale dependence of reaction in porous media [M].Advances in Water Resources. Volume 29, Issue 1, January 2006:62-71.

8. Biswal B.M, Hilfer R. Quantirtative analysis of experimental and synthetic microstructures for sedimentary rocks[J]. Physica A, 1999, 273(324): 452-475.

9. B.Straughan. Structure of the dependence of Darcy and Forchheimer coefficients on porosity [J].International Journal of Engineering Science. 2010,48(11):1610-1621.

10. A.K. Singh, R. Kumar, etc. Unsteady hydromagnetic convective flow in a vertical channel using Darcy-brinkman-forchheimer extended model with heat generation [J].International Journal of Heat and Mass Transfer. 2011,54(25-26): 5633-5642.

11. M.B. arah, A Hassairi. On the dirichlet distributions on symmetric matrices[J].journal of statistical planning and inference, 2009,139(8):2559-2570.

12. Giovanni valente John von Neumann's mathematical "Utopia" in quantum theory[J].Studies In History and Philosophy of Modern Physics, 2008,39(4): 860-871.

13. J. G. Wang. A homogenization theory for geomaterials: nonlinear effect and water flow [D]. Nagoya: Nagoya University (Japan), 1996.

14. J. G. Wang. Numerical solutions for flow in porous media [J]. Int. J. Numer. Anal.Meth. Geomech., 2003; 27:565-583. 